\title{
Retzius-sparing Robotic-assisted Radical Prostatectomy Associated with Less Bladder Neck Descent and Better Early Continence Outcome
}

\author{
LI-WEN CHANG ${ }^{1}$, SHENG-CHUN HUNG $^{1,2}$, JU-CHUAN HU $^{1}$ and KUN-YUAN CHIU ${ }^{1}$ \\ ${ }^{1}$ Division of Urology, Department of Surgery, Taichung Veterans General Hospital, Taichung, Taiwan, R.O.C.; \\ ${ }^{2}$ Institute of Medicine, Chung Shan Medical University, Taichung, Taiwan, R.O.C.
}

\begin{abstract}
Background/Aim: We attempted to evaluate the association of early continence outcome of Retzius-sparing robotic assisted radical prostatectomy (RARP) and bladder neck descent with postoperative cystography. Patients and Methods: From November 2014 to December 2015, 30 patients with prostate cancer who received Retzius-sparing $L R P / R A R P$ were compared to 30 patients that received retropubic approach $R A R P$ with propensity score matching analysis. Cystogram was used to evaluate the bladder neck descent using the bladder neck to pubic symphysis (BNPS) ratio and the continence state was evaluated. Results: A total 60 patients were included 30 of which had received Retziussparing and 30 the retropubic approach, using propensity score matching analysis. There was no difference in age, prostate size, pathology $T$ stage and PSA among groups. $B N P S$ ratio is significantly low in the Retzius-sparing group $(0.25 \pm 0.10 v s .0 .46 \pm 0.14, p=0.000)$. Early continence was improved in the Retzius-sparing group and early continence within the first week was $73.3 \%$ compared to $26.7 \%$ $(p=0.000)$, but no difference was seen at one year $(100.0 \%$ vs. $93.30 \%, p=0.150$ ). The independent risk factors affecting post-operative continence were age and approach. The association between less bladder neck descent as BNP ratio and continence was also confirmed (HR=0.048, 95\%CI=0.005-0.420, $p=0.006)$. Conclusion: With the advantage of more normal pelvic anatomy preserved in
\end{abstract}

This article is freely accessible online.

Correspondence to: Kun-Yuan Chiu, Division of Urology, Department of Surgery, Taichung Veterans General Hospital, Postal code No. 1650, Sec. 4, Taiwan Boulevard, Taichung, Taiwan, R.O.C. Tel: +886 423741215 \#5121, Fax: +886 423593160 \#5121, e-mail: chiu37782002@yahoo.com

Key Words: Cystogram, continence, radical prostatectomy, Retziusspairng.
Retzius-sparing RARP, it was associated with less bladder neck descent and better early continence outcome. Age was also an independent risk factor for postoperative continence.

Laparoscopic radical prostatectomy (LRP) was first introduced at 1991 (1), and since robotic-assisted radical prostatectomy (RARP) was first designed and established in 2000 , it has become more widespread as the standard treatment for clinically localized prostate cancer (2). Despite the advancements in oncological control and survival benefit, urinary incontinence is still a bothersome symptom in patients receiving radical prostatectomy that affect quality of life in the first year after operation. Based on the evaluation and current knowledge of the surgical anatomy, the established goal of surgery is to preserve as much continence components such as the endopelvic fascia, arcus tendineus, puboprostatic ligament, Santorini plexus and neurovascular bundle, that may reinforce the continence benefit (3).

Tracing back to the history of open surgery, perineal radical prostatectomy was first described by Young in 1905, with the better preservation of pelvic anatomy and no need to enter peritoneal cavity compared with radical retropubic prostatectomy described by Millin in 1947 (4). One of the disadvantages of this technique is that pelvic lymph node dissection cannot be performed. In the era of minimally invasive surgery, the techniques, whether laparoscopic, robotic-assisted, transperitoneal or retroperitoneal, all would take the retropubic route, the Retzius space would be opened and the bladder would be dropped (2). Despite this being the most familiar method in our daily practice with excellent functional results and an achieved continence rate of almost $62.5 \%$ at one month and $97.5 \%$ at one year (5), many patients still suffer from incontinence in the first week.

In 2010, Galfano et al. first described a novel technique of avoidance of all the anatomical structures related to continence and going directly through the Douglas space, the so-called Retzius-sparing prostatectomy (6). After opening Denonvilliers' fascia, the prostate gland would be posteriorly removed in a 
completely intrafascial plane without damage to the Santorini plexus, puboprostatic ligament, arcus tendineus and the levator ani muscle (6). Excellent results in the first 200 patients were further reported by the same surgical group with a 90-92\% immediate continence rate and a $96 \%$ one-year continence rate (7). After the learning curve, this surgical approach became established, and we adopt the same route not only in RARP but also in LRP in our clinical practice.

Considering the descent of the bladder neck after retropubic radical prostatectomy leading to an alteration in force transmission during stress and ultimately impairing normal continence (3), the location of the bladder neck may be evaluated by cystogram. The efficacy of the bladder neck to pubic symphysis (BNPS) ratio in predicting postoperative continence after radical prostatectomy was described and the lower BNPS ratio was associated with a better early continence outcome (8).

In this study, we attempted to evaluate the association of early continence outcome of Retzius-sparing robotic assisted radical prostatectomy (RARP) and bladder neck descent with postoperative cystography.

\section{Patients and Methods}

From November 2014 to March 2016, 30 patients received Retziussparing RARP at our institute by a single surgeon. Propensity score and matching analysis was used and 30 patients that had received retropubic approach RARP chosen from 150 patients receiving RARP by the same surgeon. To develop our propensity score, we used age, prostate size, and PSA. Matching was performed based on nearest-neighbor matching using a caliper width of 0.2 for the propensity score. Retropubic prostatectomy was the most familiar route at our institute and the procedure was the same as previously described (9). That is, mobilized bilateral seminal vesicle through recto-vesical pouch, dropped the bladder and entering Retzius space, bladder neck dissection with dorsal venous plexus control and dissection of prostate apex. Two set continuous running suture was used for tension free vesicourethral anastomosis.

The approved method of Retzius-sparing prostatectomy was first reported by Galfano et al. (6) and further confirmed by Lim et al. (10). In brief, the patient was put into a steep Trendelenburg position, and a $0^{\circ}$ lens was used. A horizontal incision was made at the recto-vesical pouch (pouch of Douglas), and the bilateral seminal vesicles were mobilized with clip ligation of the vas deferens. While lifting the bilateral seminal vesicles, the avascular plane between Denonvilliers' fascia and the prostatic fascia was entered near the prostatic apex, thus preserving the neurovascular bundle. If we wanted to perform an extrafascial dissection in advanced disease, Denonvilliers' fascia would be left on the prostate side to achieve maximal excision.

Then, a lateral dissection would be performed with traction and countertraction of the seminal vesicles and vas deferens, dedicated ligation of the pedicle vessels and dissection on the intrafascial/interfascial/extrafascial planes, depending on the risk of cancer and the level of neurovascular bundle preserving. The dissection would be continued nearly to the prostatic apex and dorsal venous complex (DVC).
After drawing out all of the urine in the bladder, the assistant would retract the prostate downward at the bilateral seminal vesicles and the operator would tilt the bladder upward, the bladder neck would be recognized, and the incision could be performed easily. Circum-apical dissection with a cold knife and maximal preservation of the urethra would be subsequently performed, as demonstrated in Figure 1.

After the removal of the specimens, two sets of absorbable barbed sutures (3-0 V-lock ${ }^{\mathrm{TM}}$, Covidien) would be used for vesicourethral anastomosis, beginning at 12 o'clock, with one clockwise and one counterclockwise continuous suture until 6 o'clock. The peritoneum would be closed with continuous sutures after the whole procedure was finished.

A cystogram would be done 3 days after the operation, and if there was no evidence of leakage, the Foley catheter would be removed at day 4 . The bladder neck would be located and measured as previously described by Olgin et al. (8). That is, the ratio would be calculated by measuring the distance from the superior edge of the pubic symphysis to the bladder neck and divided by the total pubic symphysis height; this so-called bladder neck to pubic symphysis (BNPS) ratio has proven value in the prediction of continence (8) (Figure 2).

Patients would be back to the clinic one week after operation and under a three monthly follow up for at least one year. Continence was defined as pad-free or only one safety pad. Surgical margin was evaluated using whole mount section and biochemical failure was defined as PSA value measured $\geq 0.2 \mathrm{ng} / \mathrm{mL}$.

Patient characteristics are shown in Table I. Kaplan-Meier survival curve estimates and log-rank tests were used to test the association between continence and operative approach. The univariate and multivariate analyses with the Cox proportional hazards model were used to predict the factors associated with continence recovery. The variables with $p$-values $<0.1$ in the univariate analysis were checked with the multivariate model, and statistical significance was accepted at $p<0.05$.

All statistical tests were carried out using IBM SPSS version 22 for Windows (SPSS, Chicago, IL, USA), with $p$-values $<0.05$ being considered statistically significant.

\section{Results}

A total of 60 patients were included with 30 receiving the Retzius-sparing and 30 the retropubic approach, using propensity score matching analysis. There was no difference between the two groups considering age, prostate size, pathology $\mathrm{T}$ stage, PSA and nerve sparing. The operation time was longer in the Retzius-sparing group but the difference was not statistically significant $(214.00 \pm 48.80 \mathrm{vs}$. $211.83 \pm 42.97, p=0.542$ ), while the amount of estimated blood loss (EBL) was relatively high in the retropubic group $(268.33 \pm 274.96 v s .149 .52 \pm 108.67, p=0.033)$. One patient in the retropubic group and 2 patients in the Retzius-sparing group suffered from urine leakage $(p=0.554)$, and all of the conditions resolved during follow-up.

Oncology result was also recorded, while the surgical margin involved in the Retzius sparing group was 9 patients (23.3\%) compared to 8 patients in retropubic prostatectomy group $(26.7 \%, p=0.261)$. Biochemical recurrence rate at one year did not appear to be different among the two groups, 


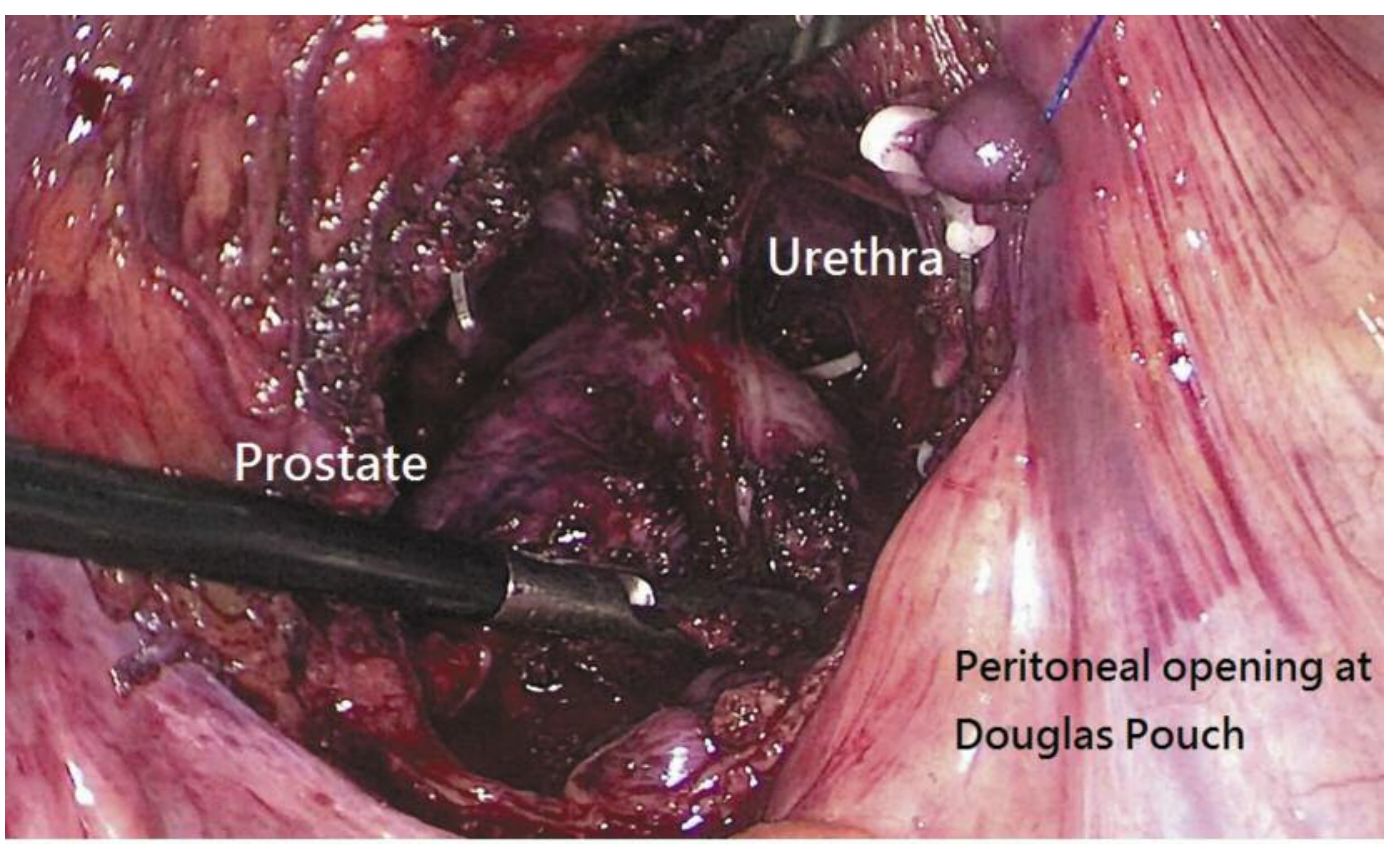

Figure 1. Figure shows the apical dissection of porstate at Retzius-sparing LRP. In the opening of peritoneal at Cau-de-sac, bilateral neurovascular bundle preservation and interfascia dissection was performed.Prostate was pull downward at seminal vesical by assistant to exposed the prostate apex.

Table I. Clinical characteristics of the patients receive retropubic approach prostatectomy and Retzius-sparing prostatectomy.

\begin{tabular}{|c|c|c|c|c|c|c|c|}
\hline \multirow[b]{2}{*}{ Age } & \multicolumn{2}{|c|}{ Retropubic approach $(\mathrm{n}=30)$} & \multicolumn{2}{|c|}{ Retzius-sparing $(\mathrm{n}=30)$} & \multicolumn{2}{|c|}{ All } & \multirow{2}{*}{$\begin{array}{c}p \text {-Value } \\
0.154\end{array}$} \\
\hline & 67.52 & \pm 6.825 & 64.39 & \pm 6.623 & 66.22 & \pm 6.810 & \\
\hline Size & 41.33 & \pm 14.80 & 40.11 & \pm 18.02 & 40.54 & \pm 16.42 & 0.671 \\
\hline PSA & 12.24 & \pm 7.79 & 18.24 & \pm 19.21 & 15.22 & \pm 14.91 & 0.130 \\
\hline Pathology & & & & & & & 0.795 \\
\hline pT2 & 17 & $56.7 \%$ & 16 & $53.3 \%$ & 33 & $55.0 \%$ & \\
\hline pT3 & 13 & $43.3 \%$ & 14 & $46.7 \%$ & 27 & $45.0 \%$ & \\
\hline Operation time & 214.00 & \pm 48.80 & 211.83 & \pm 42.97 & 213.92 & \pm 68.82 & 0.542 \\
\hline Uni Nerve spare & 11 & $36.7 \%$ & 12 & $40.0 \%$ & 23 & $38.3 \%$ & 0.791 \\
\hline Bil Nerve spare & 6 & $20.0 \%$ & 7 & $23.3 \%$ & 13 & $21.7 \%$ & 0.754 \\
\hline EBL & 268.33 & \pm 274.96 & 149.52 & \pm 108.67 & 205.22 & \pm 215.42 & $0.033^{*}$ \\
\hline Surgical Margin involved & 8 & $26.7 \%$ & 7 & $23.3 \%$ & 15 & $25.0 \%$ & 0.621 \\
\hline BNPS ratio & 0.46 & \pm 0.14 & 0.25 & \pm 0.10 & 0.35 & \pm 0.08 & $0.000 *$ \\
\hline Urine leakage & 1 & $3.3 \%$ & 2 & $6.7 \%$ & 3 & $5.0 \%$ & 0.554 \\
\hline $\mathrm{BCR}$ at one year & 5 & $16.7 \%$ & 4 & $13.3 \%$ & 9 & $15.0 \%$ & 0.718 \\
\hline Immediate continence & 8 & $26.7 \%$ & 22 & $73.3 \%$ & 30 & $50.0 \%$ & $0.000 *$ \\
\hline One year Continence & 28 & $93.3 \%$ & 30 & $100.0 \%$ & 58 & $96.7 \%$ & 0.150 \\
\hline Time to Continence(weeks) & 19.60 & \pm 17.85 & 4.20 & \pm 9.46 & 11.90 & \pm 16.15 & $0.000 *$ \\
\hline
\end{tabular}

Continous variable analysis use Student's $t$-test, mean \pm SD. Categorical variables analysis use Pearson Chi-Square test, n (\%). RARP: robotic assisted radical prostatectomy; LRP: laparoscopic radical prostatectomy; EBL: estimated blood loss; BNPS ration: bladder neck to pubic symphysis; BCR: biochemical recurrence.

with $13.3 \%$ in Retzius sparing group and $16.7 \%$ in retropubic approach group $(p=0.718)$.

Under the cystogram evaluation, the BNPS ratio appeared relatively low in the Retzius-sparing group
$(0.46 \pm 0.14 v s .0 .26 \pm 0.11, p=0.000)$, and this result implies that bladder neck descent was less in the Retzius-sparing group. Immediate continence within 7 days was more common in the Retzius-sparing group $(n=22,73.3 \%)$, while 


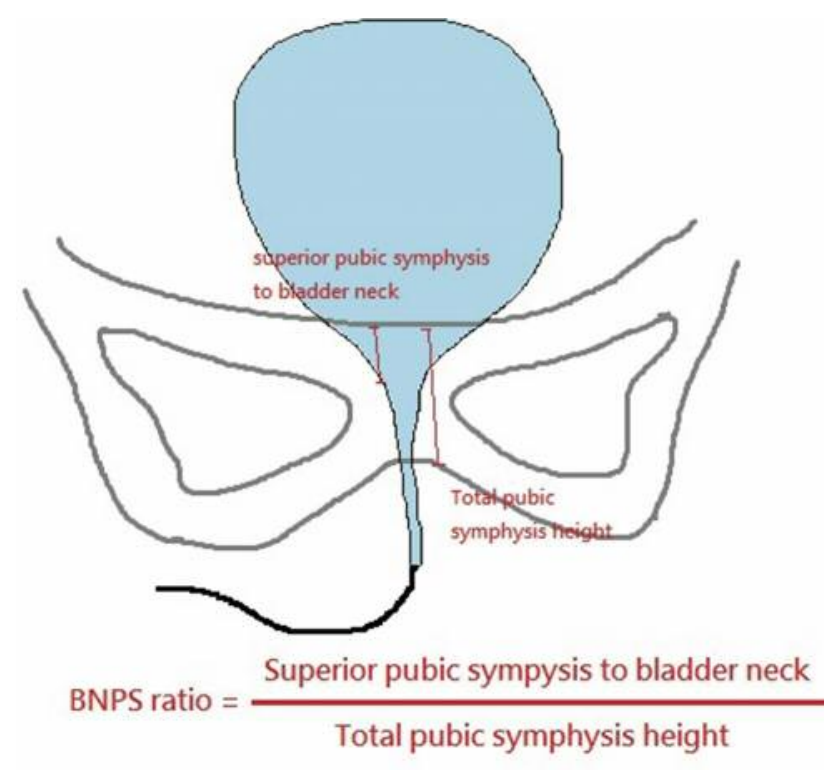

Figure 2. The ratio would be calculated by measuring the distance from the superior edge of the pubic symphysis to the bladder neck and dividing by the total pubic symphysis height; this so-called bladder neck to pubic symphysis (BNPS) ratio.

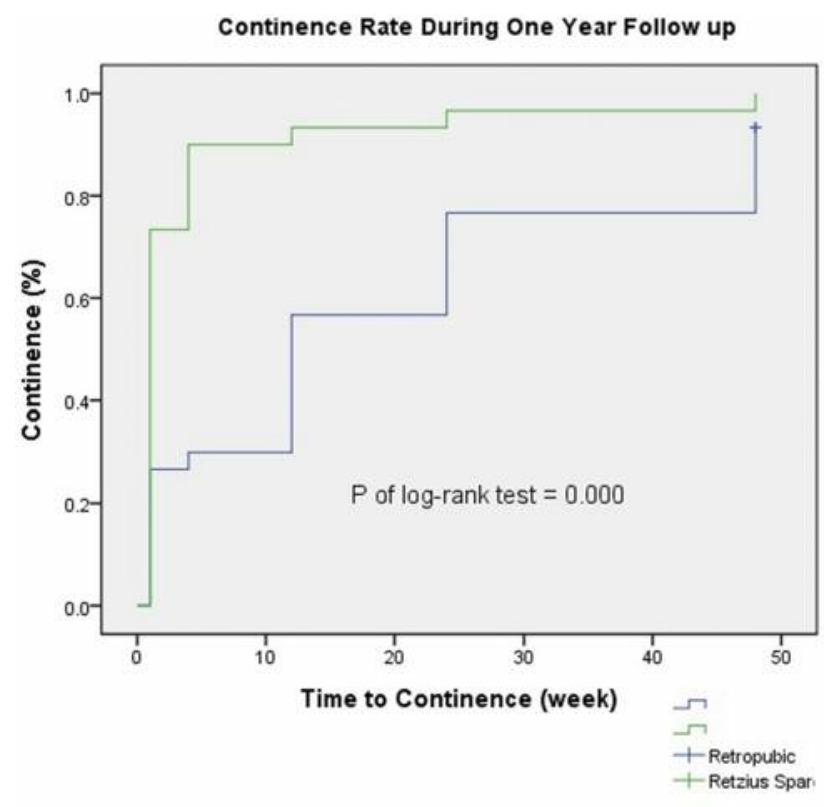

Figure 3. A Kaplan survival curve was used to evaluate the continence ratios among the two groups, and the continence rates at 1 week, 1 month, 3 months, 6 months, and 12 months were 26.7\%, 30.0\%, $56.7 \%, 76.7 \%$, and $93.3 \%$, respectively, compared with $73.3 \%, 91.0 \%$, $94.2 \%, 97.7 \%$, and $100 \%$, respectively. The p-value of log-rank test among the two groups was 0.000 .

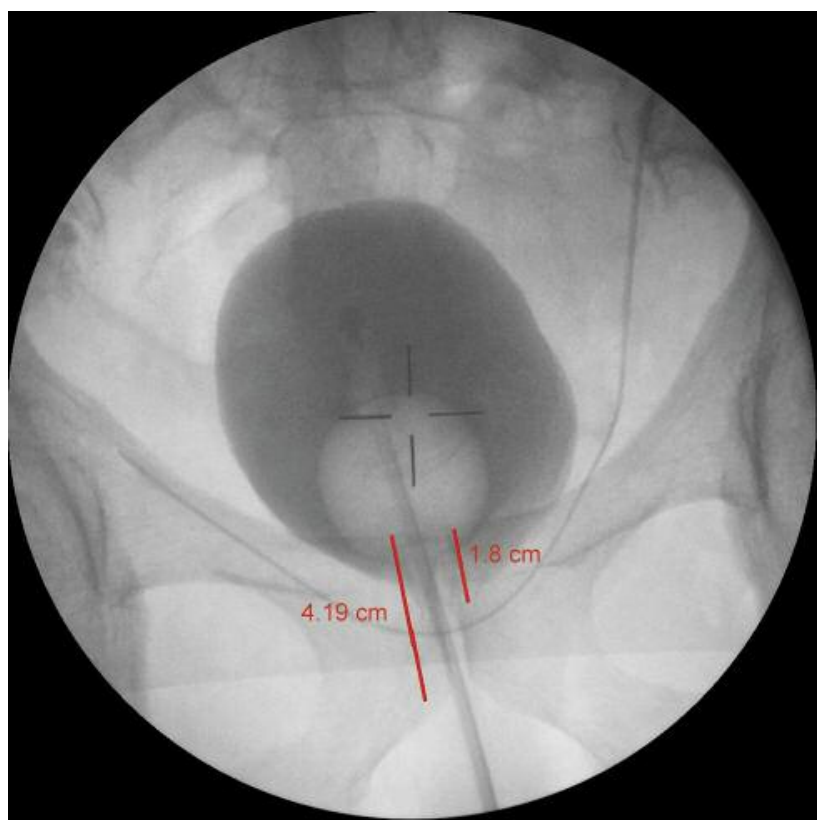

Figure 4. A 68-year-old male patient with pathology T3 prostate cancer, measure about $40 \mathrm{gm}, P S A 9.63 \mathrm{ng} / \mathrm{ml}$, received a retropubic approach $R A R P$. The operation time was $175 \mathrm{~min}$, and the EBL was $100 \mathrm{ml}$. The $B N P S$ ratio was 0.43 , and the patient achieved continence at the sixth month after Foley removal.

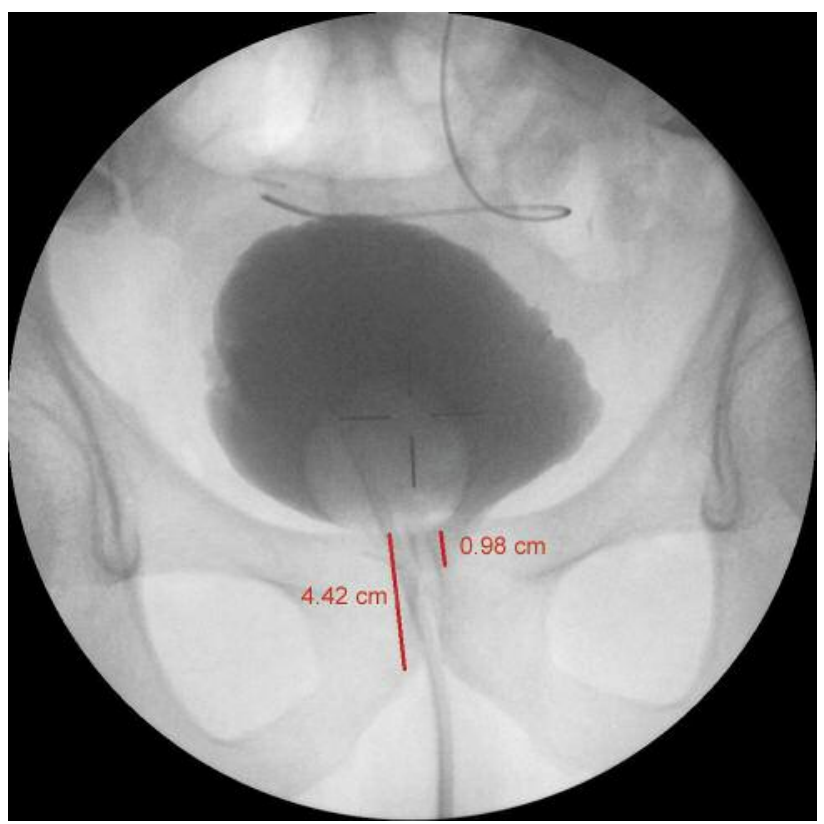

Figure 5. A 58-year-old male patient with pathology $T 2$ prostate cancer, measure about $60 \mathrm{gm}$, PSA $5.04 \mathrm{ng} / \mathrm{ml}$, received a Retzius-sparing RARP. The operation time was $168 \mathrm{~min}$, and the EBL was $80 \mathrm{ml}$. The $B N P S$ ratio was 0.22 , and the patient achieved immediate continence one week after Foley removal. 
Table II. Univariate and Multivariate Cox proportional Hazard Regression Analysis for factors associated with continence recovery.

\begin{tabular}{|c|c|c|c|c|}
\hline \multirow[b]{2}{*}{ Covariate } & \multicolumn{2}{|c|}{ Univariate } & \multicolumn{2}{|c|}{ Multivariate } \\
\hline & HR (95\%CI) & $p$-Value & HR $(95 \% \mathrm{CI})$ & $p$-Value \\
\hline Age & $0.931(0.893-0.971)$ & $0.001 *$ & $0.933(0.894-0.983)$ & $0.001 *$ \\
\hline Size & $0.994(0.977-1.010)$ & 0.442 & & \\
\hline Pathology T stage (2/3) & $0.668(0.392-1.137)$ & 0.137 & & \\
\hline PSA & $1.004(0.984-1.023)$ & 0.716 & & \\
\hline RARP/LRP & $1.244(0.716-2.161)$ & 0.439 & & \\
\hline Retzius/retropubic approach & $2.463(1.397-4.343)$ & $0.002 *$ & $2.461(1.362-4.438)$ & $0.003 *$ \\
\hline Operation time & $0.998(0.995-1.002)$ & 0.370 & & \\
\hline Nerve spare (Bil/Uni/No) & $1.172(0.591-2.323)$ & 0.650 & & \\
\hline EBL & $0.999(0.998-1.001)$ & 0.314 & & \\
\hline BNPS ratio & $0.030(0.004-0.245)$ & $0.001 *$ & $0.048(0.005-0.420)$ & $0.006^{*}$ \\
\hline Urine leakage & $0.526(0.163-1.699)$ & 0.283 & & \\
\hline
\end{tabular}

HR: Harzard ratio; CI; confidence interval; LRP: laparoscopic radical prostatectomy; RARP: robotic assisted radical prostatectomy; EBL: estimated blood loss; BNPS ratio: bladder neck to pubic symphysis; $p<0.05$ statistical significance*.

there were only 8 patients in the retropubic group $(26.7 \%$, $p=0.000)$. There was no difference in the one-year continence between the two groups $(100.0 \%$ vs. $93.3 \%$, $p=0.150)$.

A Kaplan survival curve was used to evaluate the continence ratios among the two groups in Figure 3, and the continence rates at 1 week, 1 month, 3 months, 6 months, and 12 months were $26.7 \%, 30.0 \%, 56.7 \%, 76.7 \%$, and $93.3 \%$, respectively, compared with $73.3 \%, 91.0 \%, 94.2 \%$, $97.7 \%$, and $100.0 \%$, respectively $(p=0.000)$. The time to continence also appeared sooner in the Retzius group $(4.1 \pm 9.52$ weeks $)$ than in the retropubic approach group $(19.60 \pm 17.85$ weeks, $p=0.000)$.

Regarding the risk factors of incontinence in Table II, compared to the retropubic approach, the Retzius-sparing approach achieved better continence result $(\mathrm{HR}=2.461$, $95 \% \mathrm{CI}=1.362-4.438)$. Age also proved to be an independent factor associated with continence $(\mathrm{HR}=0.933,95 \% \mathrm{CI}=0.894$ $0.983)$. The prediction efficacy of BNPS ratio was also confirmed in thre multivariate analysis ( $\mathrm{HR}=0.048$, 95\% CI=0.005-0.420).

Figure 4 demonstrates a patient with pathology T3 prostate cancer, measuring approximately $40 \mathrm{gm}$, that received the retropubic approach RARP. The BNPS ratio showed 0.43 , and the patient achieved continence at the sixth month. Figure 5 shows a patient with pathology T2 prostate cancer, measuring approximately $45 \mathrm{~cm}$, that received the Retzius-sparing RARP. The cystogram shows less bladder neck descent, and the BNPS ratio was relatively low (0.22) in this patient. He also achieved continence within the first week after Foley catheter removal.

\section{Discussion}

In the present study, we retrospectively reviewed our patients with clinical localized prostate cancer receiving RARP with a propensity scoring matched method, and revealed that the Retzius-sparing approach is associated with less bladder neck descent and better early continence outcome.

The Retzius-sparing RARP was first reported in 2010 with the benefits of (1) the possibility of completely intrafascial prostatectomies with preservation of Aphrodite's veil; (2) the avoidance of the Santorini plexus with less blood loss; (3) the sparing of the pubourethral ligaments and accessory pudendal arteries, which may be related to continence and potency; and (4) only making a small opening in the peritoneum at the Douglas pouch, which reduced trauma or adhesions (6). Lim et al. also described this technique as a way to "combine the benefit of perineal radical prostatectomy with spares the Retzius space and dorsal vein and the benefit of retropubic radical prostatectomy with preservation of endopelvic fascia and pelvic floor musculature" (10). With the reported excellent early continence outcome (7), this technique has also been adopted in our practice since 2014. It appears more difficult using the laparoscopic method because the robotic system, with its one assistance arm, allows a more dedicated dissection and reconstruction to be performed in a more comfortable position. Despite the challenges, our previously published video also demonstrated the adaption and feasibility of this technique in the laparoscopic approach (11).

The dramatic result in the first large series of Retziussparing RARP by Galfano et al. had a $40 \%$ rate of intercourse at 1 month and a $>90 \%$ immediate continence 
after surgery (7). Lim et al. also reported similar results, with $92 \%$ continence and $70 \%$ being completely dry at one month; this encouraging result was not observed in the conventional approach group previously (10). In our patient group of Retzius-sparing approach, a 73.3\% immediate continence rate was observed within the first week, and $91.00 \%$ continence was observed at one month, whereas in the retropubic group, theywere $26.7 \%$ and $30.0 \%$ respectively $(p=0.000)$, which was the same as the previous reported study. That may be due to less damage to the components such as the levator ani muscles, the pubourethral ligaments, and the puboprostatic ligaments, which suspend the floor, may prevent bladder descent and contribute to less uretheral hypermobility and early continence (12).

The BNPS ratio in the cystogram has been used to define the bladder neck location and to predict postoperative continence. Olgin et al., first descript the measured method with BNPS in prediction of postoperative continence in patient received radical prostatectomy and reported the continence rate of the lowes BNPS (mean 0.16), intermediate BNPS (mean 0.41) and high BNPS (mean 0.70) group were $80.3 \%, 72.7 \%$ and $59.7 \%$, respectively (8). Our results also confirm the predictive value of the cystogram in the unimultivariate analysis model and this may be used as a tool to predict functional outcome or evaluated anatomic condition of pelvic floor. Also, the BNPS ratios of the Retzius-sparing were significantly lower than the retropubic RARP in our surgical group. Figures 4 and 5 further demonstrate the different results among these two approaches in the illustration.

Prostate size was not an independent risk factor of incontinence after operation in our series, and this result was also observed in the Retzius-sparing RARP group by Lim et al. (13). They claimed that large prostate size appears to be equivocally relative to oncological and continence outcomes and was not associated with a longer operative or console time, despite the increased surgical difficulty (13).

Age, as the most observed risk factor of incontinence after prostatectomy $(14,15)$, was also confirmed in our patient's group, while older age reduced the achievement of continence $(\mathrm{HR}=0.932,95 \% \mathrm{CI}=0.893-0.973)$. This may be related to the condition that, as with other muscles, the sphincter muscle deteriorates with age.

There are some limitations to our study. Small sample size and retrospective study design may be the major drawback. The improvement of potency was not reported in our literature, that is, of the majority of Asia's elderly population, they care more about continence than intercourse.

Despite the limited experience, however, it is an exciting result that this novel technique may be encountered as having an excellent functional outcome without compromising oncological control and it may be more worldwide accepted in the future.

\section{Conclusion}

With the advantage of a more normal pelvic anatomy preserved using Retzius-sparing RARP, it was associated with less bladder neck descent and a better early continence outcome. Age was also an independent risk factor for postoperative continence.

\section{Conflicts of Interest}

The contributing Authors have no conflicts of interest, including specific financial interests or relationships and affiliations relevant to the subject matter or materials discussed in the manuscript.

\section{References}

1 Schuessler WW, Schulam PG, Clayman RV and Kavoussi LR: Laparoscopic radical prostatectomy: initial short-term experience. Urol 50(6): 854-857, 1997.

2 Menon M, Shrivastava A, Tewari A, Sarle R, Hemal A, Peabody JO and Vallancien G: Laparoscopic and robot assisted radical prostatectomy: establishment of a structured program and preliminary analysis of outcomes. J Urol 168(3): 945-949, 2002.

3 Walz J, Burnett AL, Costello AJ, Eastham JA, Graefen M, Guillonneau B, Menon M, Montorsi F, Myers RP, Rocco B and Villers A: A critical analysis of the current knowledge of surgical anatomy related to optimization of cancer control and preservation of continence and erection in candidates for radical prostatectomy. Eur Urol 57(2): 179-192, 2010.

4 Lepor H: A review of surgical techniques for radical prostatectomy. Rev Urol 7(Suppl 2): S11-S17, 2005.

5 Ou YC, Yang CK, Kang HM, Chang KS, Wang J, Hung SW, Tung MC, Tewari AK and Patel VR: Pentafecta outcomes of 230 cases of robotic-assisted radical prostatectomy with bilateral neurovascular bundle preservation. Anticancer Res 35(9): 50075013, 2015.

6 Galfano A, Ascione A, Grimaldi S, Petralia G, Strada E and Bocciardi AM: A new anatomic approach for robot-assisted laparoscopic prostatectomy: a feasibility study for completely intrafascial surgery. Eur Urol 58(3): 457-461, 2010.

7 Galfano A, Di Trapani D, Sozzi F, Strada E, Petralia G and Bramerio M: Beyond the learning curve of the Retzius-sparing approach for robot-assisted laparoscopic radical prostatectomy: oncologic and functional results of the first 200 patients with $\geq 1$ year of follow-up. Eur Urol 64(6): 974-980, 2013.

8 Olgin G, Alsyouf M, Han D, Li R, Lightfoot M, Smith D, Nicolay L, Ruckle H and Baldwin DD: Postoperative cystogram findings predict incontinence following robot-assisted radical prostatectomy. J Endourol 28(12): 1460-1463, 2014.

9 Hung SC, Ou YC, Cheng CL, Hung SW, Ho HC, Chiu KY, Wang SS, Chen CS, Li JR and Yang CK: Standardized procedure of robotic assisted laparoscopic radical prostatectomy from case 1 to case 1200. Urol Sci 27(4): 199-207, 2016.

10 Lim SK, Kim KH, Shin TY, Han WK, Chung BH, Hong SJ, Choi YD and Rha KH: Retzius-sparing robot-assisted laparoscopic radical prostatectomy: combining the best of retropubic and perineal approaches. BJU Int 114: 236-244, 2014. 
$11 \mathrm{Hu}$ JC and Chiu KY: Laparoscopic Retzius-Sparing radical prostatectomy: Single surgeon experiences. J Endourol Videourology 31, 2017. https://doi.org/10.1089/vid.2016.0044. [Epub ahead of print].

12 Dev HS, Sooriakumaran P, Srivastava A and Tewari AK: Optimizing radical prostatectomy for the early recovery of urinary continence. Nat Rev Urol 9(4): 189-195, 2012.

13 Santok GD, Abdel Raheem A, Kim LH, Chang K, Lum TG, Chung BH, Choi YD and Rha KH: Perioperative and short-term outcomes of Retzius-sparing robot-assisted laparoscopic radical prostatectomy stratified by gland size. BJU Int 119(1): 135-141, 2017.
14 Sacco E, Prayer-Galetti T, Pinto F, Fracalanza S, Betto G, Pagano $\mathrm{F}$ and Artibani W: Urinary incontinence after radical prostatectomy: incidence by definition, risk factors and temporal trend in a large series with a long-term follow-up. BJU Int 97(6): 1234-1241, 2006.

15 Wolin KY, Luly J, Sutcliffe S, Andriole GL and Kibel AS: Risk of urinary incontinence following prostatectomy: the role of physical activity and obesity. J Urol 183: 629-633, 2010.

Received October 9, 2017

Revised October 25, 2017

Accepted October 26, 2017 